\title{
Cyclic AMP responsive element-binding protein induces metastatic renal cell carcinoma by mediating the expression of matrix metallopeptidase- $2 / 9$ and proteins associated with epithelial-mesenchymal transition
}

\author{
XUE WANG ${ }^{1,2}$, HUI CUI ${ }^{1,2}$, ZHONGGUAN LOU ${ }^{1,2}$, \\ SHUAISHUAI HUANG ${ }^{1,2}$, YU REN ${ }^{1,2}$, PING WANG ${ }^{2,3}$ and GUOBIN WENG ${ }^{1,2}$ \\ ${ }^{1}$ Department of Urologic Surgery, Ningbo Urology and Nephrology Hospital; \\ ${ }^{2}$ Laboratory of Kidney Carcinoma, Urology and Nephrology Institute of Ningbo University, Ningbo, \\ Zhejiang 315000; ${ }^{3}$ Ningbo University School of Medicine, Ningbo, Zhejiang 315211, P.R. China
}

Received October 14, 2016; Accepted January 10, 2017

DOI: 10.3892/mmr.2017.6519

\begin{abstract}
Renal cell carcinoma (RCC) is the most frequently occurring malignancy of the kidney worldwide. Anti-angiogenic targeted therapies inhibit the progression of RCC, however, limited effects on the invasion or metastasis of tumor cells have been observed. Cyclic AMP responsive element-binding protein (CREB) is a serine/threonine kinase that has been implicated in the regulation of cell proliferation, apoptosis, cycle progression and metastasis, amongst others. Our previous research demonstrated that phosphorylated CREB (pCREB) was upregulated in human renal cancer cell lines and tissues, and decreased pCREB at the Ser133 site inhibited the growth and metastatic activity of OS-RC-2 cells. However, the role of CREB in RCC metastasis requires further investigation. Thus, the present study further investigated the role of CREB in RCC metastasis. The present study demonstrated that knockdown of CREB using small interfering RNA (siRNA) that targeted CREB (siCREB) significantly inhibited the migration and invasion of 786-O and OS-RC-2 cells, however, the opposite effect was observed in ACHN cells. In addition, knockdown of CREB suppressed the expression of matrix metallopeptidase (MMP)-2/9 and proteins associated with epithelial-mesenchymal transition (EMT) in 786-O and OS-RC-2 cells, and promoted expression in ACHN cells. Furthermore, the chromatin immunoprecipitation assay indicated that pCREB (Ser133)
\end{abstract}

Correspondence to: Professor Guobin Weng, Department of Urologic Surgery, Ningbo Urology and Nephrology Hospital, 1 Qianhe Road, Yinzhou, Ningbo, Zhejiang 315000, P.R. China E-mail: nbuurology@126.com

Key words: cyclic AMP responsive element-binding protein, matrix metallopeptidase-2/9, epithelial-mesenchymal transition, renal cell carcinoma metastasis had a direct interaction with the fibronectin promoter, however, pCREB (Ser133) did not target the vimentin promoter in RCC. Therefore, the results of the present study indicate that CREB regulated metastatic RCC by mediating the expression of MMP-2/9 and EMT-associated proteins, however, CREB-mediated MMP-2/9 and EMT-associated protein expression may be induced by different pathways in different RCC cells.

\section{Introduction}

Renal carcinoma is the 13th most common cancer worldwide (1). There were $\sim 61,560$ new cases of renal cancer and 14,080 deaths due to renal cancer in 2015 in the United States according to statistics by Siegel et al (2). Renal cell carcinoma (RCC) accounts for $\sim 3 \%$ of all malignancies in adults (3), Clear cell renal cell carcinoma (CCRCC) is the most common type of RCC, which is responsible for $\sim 75 \%$ of all RCC cases worldwide (4). Radical nephrectomy is effective for curing early and local RCC, however, the response of advanced or metastatic RCC to chemotherapy or radiotherapy is limited and individuals have poor prognosis with an average survival of only 6-12 months from the time of diagnosis $(5,6)$. Therefore, it is important to improve the understanding of the pathogenesis of aggressive RCC and to select biomarkers in order to develop effective strategies for the prevention and treatment of RCC.

Cyclic AMP (cAMP) responsive element-binding protein (CREB) is a transcription factor that has a critical role in the regulation of tumorigenesis $(7,8)$. In response to various signals, on binding to the cAMP-responsive element (CRE) sequence (TGACGTCA), phosphorylation of CREB (pCREB) at the Ser133 residue is activated by a number of kinases (9). Activated CREB subsequently regulates various cell functions by enhancing the expression of target genes (10-12). Several studies have demonstrated that overexpression of pCREB promotes tumorigenesis in various cancer tissues, including acute myeloid leukemia $(13,14)$, non-small cell lung carcinoma $(15,16)$, breast $(17,18)$, melanoma (19) and hepatocellular 
cancers (20). However, the role of CREB in RCC requires further investigation.

Our previous study demonstrated that pCREB was upregulated in human renal cancer cell lines and tissues. Lentiviral vector production was used to knockdown the expression of pCREB in OS-RC- 2 cells and the results demonstrated that decreasing the level of pCREB inhibited the growth and metastasis of OS-RC-2 cells (21). However, although the results are promising, further studies are needed to confirm this. Therefore, the present study investigated, in multiple human renal cell carcinoma cell lines, whether pCREB regulates metastasis via epithelial-mesenchymal transition (EMT)-associated proteins and matrix metallopeptidase (MMP)-2/9. The current study additionally aimed to determine whether the human fibronectin promoter contains a functional CRE that activates fibronectin transcription upon pCREB binding at the Ser133 site.

\section{Materials and methods}

Cell culture. The human renal cancer cell lines (786-Oand ACHN) and an immortalized proximal tubule epithelial cell line (HK-2) were obtained from American Type Culture Collection (Manassas, VA, USA). The human renal cancer cell line OS-RC-2 was obtained from the Cell Bank of the Chinese Academy of Sciences (Shanghai, China). The 786-O and OS-RC-2 cell lines were maintained in RPMI-1640 (HyClone; GE Healthcare Life Sciences, Logan, UT, USA), the ACHN and HK-2 cell lines were maintained in Dulbecco's modified Eagle's medium (HyClone; GE Healthcare Life Sciences). The medium was supplemented with $10 \%$ fetal bovine serum (FBS; Shanghai ExCell Biology, Inc., Shanghai, China). All cells were cultured in a humidified atmosphere at $37^{\circ} \mathrm{C}$ in $5 \% \mathrm{CO}_{2}(17)$.

Transfection of cell lines. Small interfering RNA (siRNA) for CREB (siCREB; 5'-GUCUCCACA AGUCCAAAC ATT-3'; antisense, 5'-UGUUUGGACUUGUGGAGACTT-3') and siRNA for negative control (siNC; sense, 5'-UCCUCC GAACGUGUCACGUTT-3'; antisense, 5'-ACGUGACAC GUUCGGAGAATT-3') were purchased from Shanghai GenePharma Co., Ltd (Shanghai, China). A total of $5 \times 10^{5}$ cells were seeded in 6-well plates for $24 \mathrm{~h}$ at $37^{\circ} \mathrm{C}$ and transfected with specific siCREB (100 pmol) or control siNC (100 pmol) by using Lipofectamine ${ }^{\circledR} 2000$ reagent (Invitrogen; Thermo Fisher Scientific, Inc., Waltham, MA, USA, according to the manufacturer's protocol (22).

Wound healing assay. Cells were added to a 24 well-plate at a concentration of $0.7 \times 10^{5}$ cells/well in $1 \mathrm{ml} 10 \%$ FBS-containing medium. Cells were cultured for $24 \mathrm{~h}$ and reached $70 \%$ confluence at $37^{\circ} \mathrm{C}$ in $5 \% \mathrm{CO}_{2}$. Linear scratches were made with a micropipette tip across the diameter of the well and dislodged cells were rinsed with PBS. Cell culture medium was replaced with fresh, serum-free medium. Images of the scratch were acquired as baseline and images of the same location were obtained after $24 \mathrm{~h}$. Experiments were performed in quadruplicate (23).

Cell invasion assay. The cell invasion assay was performed using BD Matrigel 24-well $8 \mu \mathrm{m}$ invasion chambers with filters coated with extracellular matrix on the upper surface (BD Biosciences, Franklin Lakes, NJ, USA). Briefly, $100 \mu \mathrm{l}$ serum-free medium containing $1 \times 10^{4}$ cells from each subgroup were added to the upper chamber. The $600 \mu 1$ medium (HyClone; GE Healthcare Life Sciences) containing 20\% FBS (Shanghai ExCell Biology, Inc.) was added to the lower chamber as a chemoattractant. Cells were allowed to invade for $24 \mathrm{~h}$ at $37^{\circ} \mathrm{C}$ in $5 \% \mathrm{CO}_{2}$. The cells in the upper chamber were removed using a cotton swab. Cells that had migrated to the bottom of the membrane were fixed and stained 30 min with $0.1 \%$ crystal violet staining solution at room temperature and were photographed from 5 different microscopic fields (Motic AE31; Motic China Group Co., Ltd., Xiamen, China). Crystal violet was removed by addition of $33 \%$ acetic acid at $200 \mu \mathrm{l} /$ well to the 96 -well plates and absorbance was measured at $570 \mathrm{~nm}$ using a SpectraMax Plus384; Molecular Devices LLC (Sunnyvale, CA, USA). Experiments were performed in triplicate (23).

Western blot analysis. For western blot analysis, $50 \mu \mathrm{g}$ of each sample were processed as described (24). CREB (catalog no. 9197), pCREB (Ser133; catalog no. 9198), N-cadherin (catalog no. 14215), E-cadherin (catalog no. 3195), $\beta$-actin (catalog no. 8457) and GAPDH (catalog no. 5174) antibodies were obtained from Cell Signaling Technology, Inc. (Danvers, MA, USA). MMP-2 (catalog no. ab7033), MMP-9 (catalog no. ab137651), vimentin (catalog no. ab8978) and fibronectin (catalog no. ab2413) antibodies were obtained from Abcam (Cambridge, UK). All primary antibodies were diluted at 1:1,000 and were used according the manufacturer's protocol. Goat anti-rabbit IgG-HRP or goat anti-mouse IgG-HRP were used (dilution 1;5,000; catalog no BA1054/BA1050; Wuhan Boster Biological Technology, Ltd., Wuhan, China) and detected by chemiluminescence. Densitometric analysis was performed by Tanon GIS version 4.1.2 software (Tanon Science and Technology Co., Ltd., Shanghai, China).

Chromatin immunoprecipitation (ChIP) assay. ChIP assays were performed using a SimpleChIP ${ }^{\circledR}$ Enzymatic Chromatin IP kit (catalog no. 9002; Cell Signaling Technology, Inc.) according to the manufacturer's protocol. IgG antibody was included in the ChIP assay kit. pCREB (Ser133) antibody (dilution 1:50; catalog no. 9198) was obtained from Cell Signaling Technology, Inc. Quantification of immunoprecipitated DNA was performed using quantitative polymerase chain reaction (qPCR) with LightCycler 480 SYBR-Green I Master (Roche Diagnostics, Indianapolis, IN, USA) according to the manufacturer's protocol. CREB binds to a target sequence termed the CRE-site and this serves as a model for the research of CREB function, and the CREB-binding protein (CRE-BP) site is the transcription factor CRE-BP binding site. The -449 to -442 bp (CRE site) fibronectin promoter sequence from the transcription start site had the following primers: 5'-CCG AAAAAAAGTTGTCTTGCCC-3' (forward); and 5'-CAG CCGACCGCGCGCCGATTGG-3' (reverse). The -731 to -722 bp (CRE-BP site) vimentin promoter sequence from the transcription start site had the following primers: 5'-TATTGC CGCCAAAGATTCTG-3' (forward); and 5'-TACCCTGGT GGAAGTCATTAAAG-3' (reverse). ChIP results were calculated and presented as a percentage relative to the input DNA by the $\Delta \Delta \mathrm{Cq}$ method $(25,26)$. 


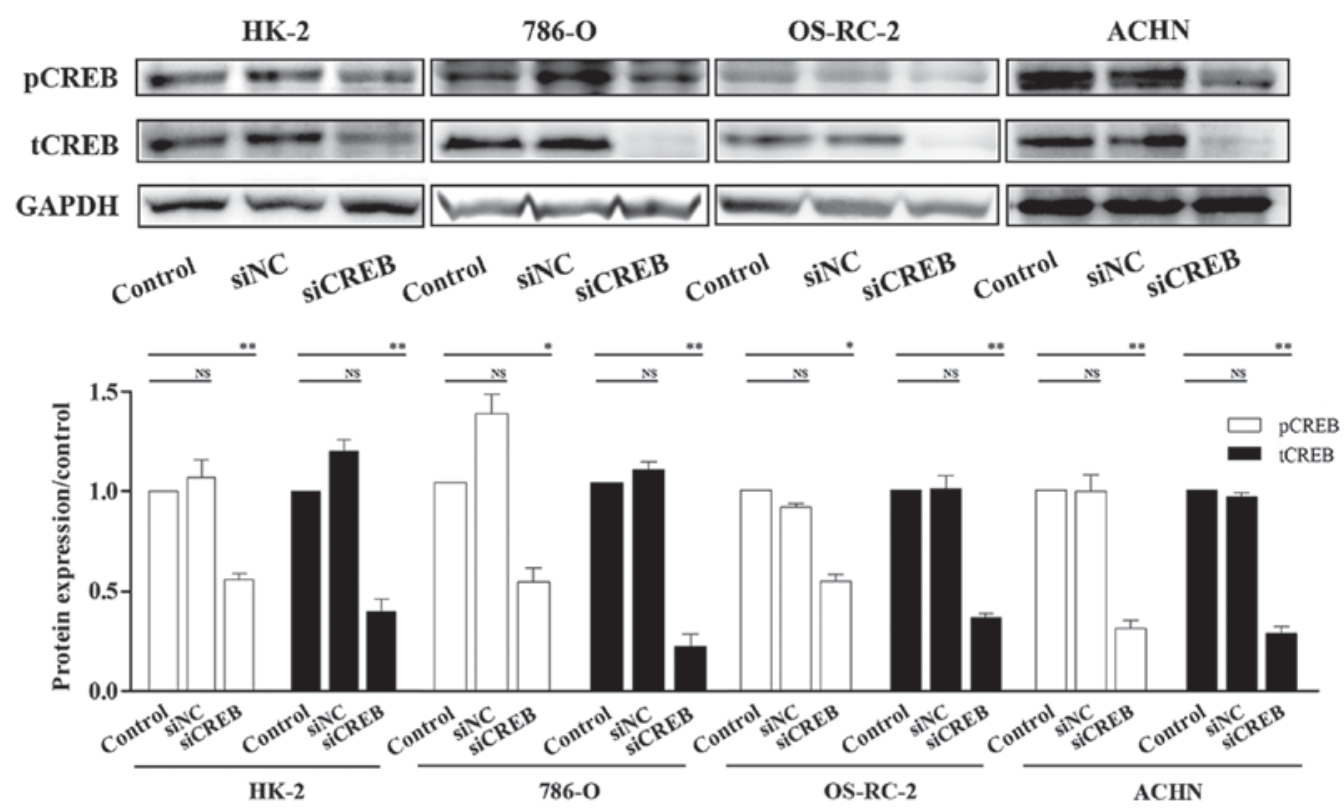

Figure 1. Transfection with siCREB significantly reduced the expression of tCREB and pCREB. Representative blots and analysis of gray value demonstrating the reduced protein level of CREB in siCREB-transfected 786-O, OS-RC-2, ACHN and HK-2 cells compared with control and siNC-transfected cells. ${ }^{*} \mathrm{P}<0.05$ and ${ }^{* *} \mathrm{P}<0.01$ vs. control. Results are presented as the mean \pm standard deviation from 3 independent experiments. siRNA, small interfering RNA; CREB, cyclic AMP responsive element-binding protein; tCREB, total CREB; pCREB, phosphorylated CREB; NC, negative control; NS, not significant.

Reverse transcription-qPCR. Using the standard TRIzol protocol (Invitrogen; Thermo Fisher Scientific, Inc.), total RNA was isolated and extracted from cells. The amounts of total RNA were quantified using spectrophotometric measurements. Using a RevertAid First Strand cDNA Synthesis Kit (K1622; Thermo Fisher Scientific, Inc.) according to the manufacturer's protocol, total RNA was reversed transcribed into cDNA. qPCR was performed using the LightCycler 480 SYBR-Green I Master (Roche Diagnostics). Each standard qPCR reaction contained the following; Reverse transcriptase product $(2 \mu \mathrm{l}) ; 0.5 \mu \mathrm{l}$ of each primer (final concentration, $0.1 \mathrm{mM}$ ); $12.5 \mu$ l SYBR-Green PCR Master Mix ${ }^{\mathrm{TM}}$ (Roche Diagnostics). The thermocycling conditions included an initial 15 min holding period at $95^{\circ} \mathrm{C}$, followed by a PCR program repeated for 50 cycles: $15 \mathrm{sec}$ at $94^{\circ} \mathrm{C}, 57 \mathrm{sec}$ at $30^{\circ} \mathrm{C}$ and $30 \mathrm{sec}$ at $70^{\circ} \mathrm{C}$. The following primers were used: MMP-2, 5'-TTGACGGTAAGGACGGACTC-3' (forward) and 5'-ACTTGCAGTACTCCCCATCG-3' (reverse); MMP-9, 5'-TTGACAGCGACAAGAAGTGG-3' (forward) and 5'-CCCTCAGTGAAGCGGTACAT-3' (reverse); GAPDH, 5'-AAGCCTGCCGGTGACTAAC-3' (forward) and 5'-GCA TCACCCGGAGGAGAAAT-3' (reverse). GAPDH was used as a normalization and other gene expression levels were analyzed using the $\Delta \Delta \mathrm{Cq}$ method (25). All samples were performed in triplicate and, for each reaction, negative controls without reverse transcriptase or RNA were performed $(12,27)$.

Statistical analysis. All experiments were repeated 3 times. Using SPSS, version 18.0 (SPSS, Inc., Chicago, IL, USA), results are presented as the mean \pm standard deviation. One-way analysis of variance and Fisher's least significant difference tests were performed to evaluate the differences between groups. $\mathrm{P}<0.05$ was considered to indicate a statistically significant difference.

\section{Results}

Effects of CREB downregulation on cell migration and invasion. In the present study, to investigate, in multiple human RCC cell lines, the functional role of the upregulation of CREB, siCREB was used to knockdown the expression of CREB in 786-O, OS-RC-2 and ACHN RCC cells, and normal HK-2 cells. To detect the effect of siCREB, the expression of CREB was investigated by western blotting. The results demonstrated that the protein expression of CREB and pCREB were significantly downregulated compared with cells transfected with siNC and control, following cell transfection with siCREB (Fig. 1). In addition, cell migration and invasion ability was analyzed using wound healing and cell invasion assays. Transfection with siCREB significantly blocked migration $(\mathrm{P}<0.01$; Fig. 2$)$ and invasion $(\mathrm{P}<0.01$; Fig. 3$)$ in $786-\mathrm{O}$ and OS-RC-2 cells compared with control, however, significantly increased migration $(\mathrm{P}<0.05$; Fig. 2$)$ and invasion $(\mathrm{P}<0.01$; Fig. 3) in ACHN cells compared with control, and demonstrated no significant effects in HK-2 cells (Figs. 2 and 3). These results indicate that CREB may contribute to the migration and invasion phenotype of RCC cells, and not HK-2 cells, however, results were not consistent between the 3 RCC cell lines.

CREB regulates the expression of $M M P-2$ and MMP-9 in $R C C$ cells. MMPs may disrupt the extracellular matrix to promote cancer cell mobility, which eventually causes metastasis $(28,29)$. Among MMPs, MMP-2 and MMP-9 are vital enzymes involved in the degradation of gelatin, collagen and laminin (30). The expression of numerous genes is activated by CREB (31). Therefore, the present study aimed to investigate whether CREB regulates RCC cell migration and invasion ability by affecting MMP-2/9 expression. As observed in the results of the wound healing and cell invasion assay, 786-O 

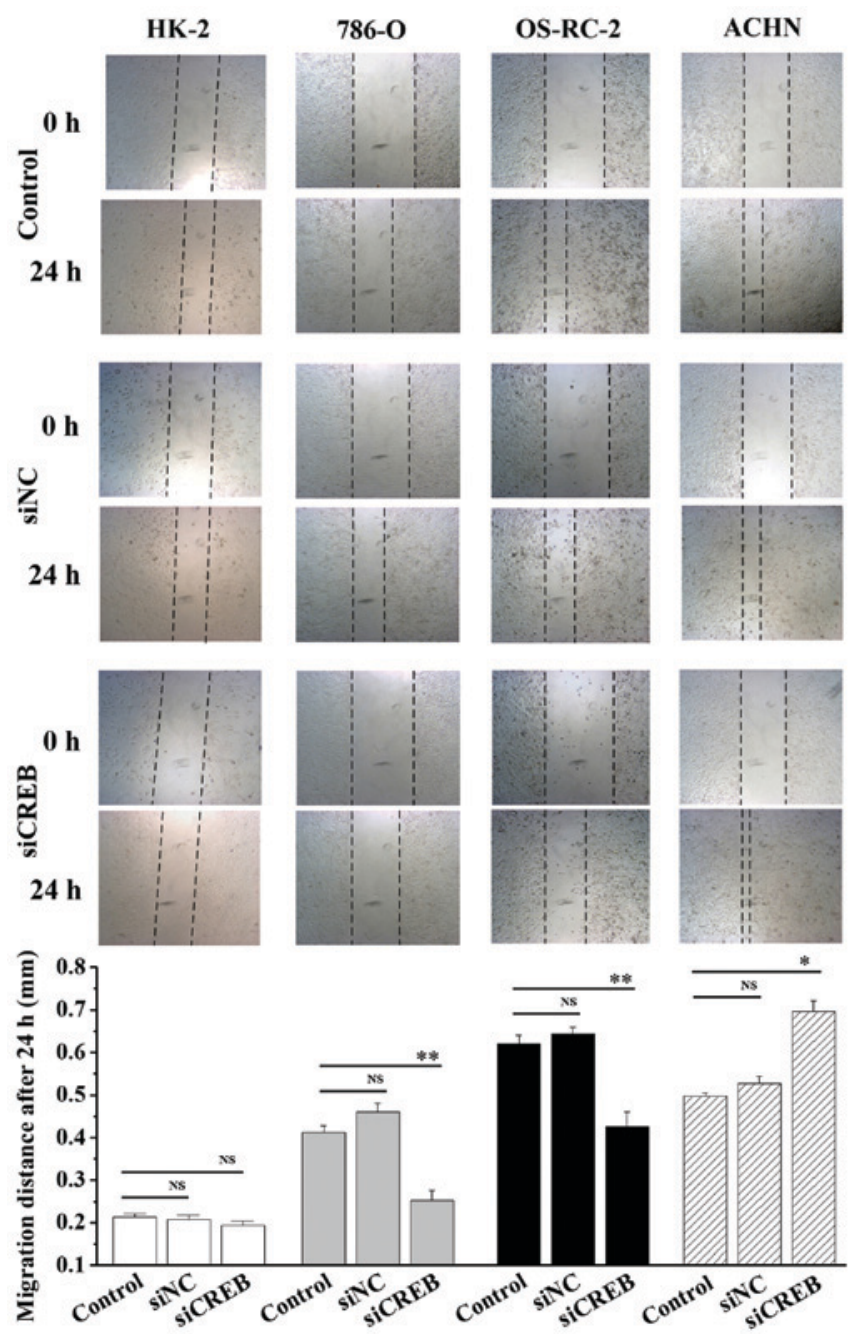

Figure 2. Effect of CREB knockdown on RCC cell migration activity in vitro. The migratory capacity of RCC cells was examined by the wound healing assay. Migratory ability in siCREB groups were significantly inhibited compared with control groups in 786-O and OS-RC-2 cells, whereas knockdown of CREB promoted migration in ACHN cells and no significant effects were observed in HK-2 cells. ${ }^{*} \mathrm{P}<0.05$ and ${ }^{* *} \mathrm{P}<0.01$ vs. control. Results are presented as the mean \pm standard deviation from 3 independent experiments. CREB, cyclic AMP responsive element-binding protein; RCC, renal cell carcinoma; siRNA, small interfering RNA; siCREB, siRNA targeting CREB; siNC, siRNA negative control; NS, not significant.

and OS-RC-2 cells, following siCREB transfection, expressed significantly lower levels of MMP-2/9 protein $(\mathrm{P}<0.01$; Fig. $4 \mathrm{~A})$ and mRNA $(\mathrm{P}<0.01$; Fig. 4B) compared with the siNC group, however, the opposite was observed in ACHN cells where protein and mRNA levels of MMP-2/9 were significantly increased compared with the siNC group ( $\mathrm{P}<0.01$; Fig. 4). Combined, the results demonstrate that $\mathrm{CREB}$ regulated the migration and invasion of the 3 types of RCC cells, however, the effects of CREB knockdown were different between the 3 RCC cell lines.

$C R E B$ expression is associated with the expression of certain EMT markers in RCC cells. The EMT is a highly conserved program necessary for orchestrating distant cell migration during embryonic development. A previous report demonstrated a critical role for EMT during the initial stages of tumorigenesis and later during tumor invasion (32). Thus, in
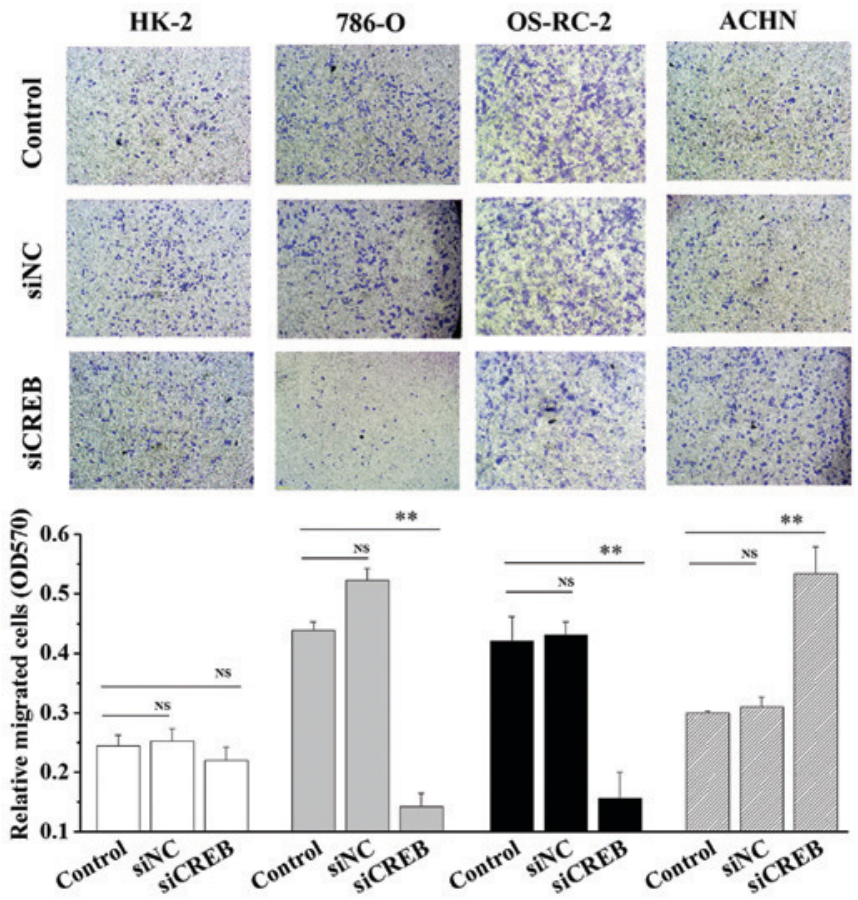

Figure 3. Effect of siCREB transfection on RCC cell invasion in vitro RCC cell invasion activity determined by cell invasion assay. Results were consistent with the results of the wound healing assay. ${ }^{*} \mathrm{P}<0.05$ and ${ }^{* *} \mathrm{P}<0.01$ vs. control. Results are presented as the mean \pm standard deviation from 3 independent experiments. CREB, cyclic AMP responsive element-binding protein; siRNA, small interfering RNA; siCREB, siRNA targeting CREB; RCC, renal cell carcinoma; siNC, siRNA negative control; OD, optical density; NS, not significant.

order to further investigate the association between CREB and EMT, markers associated with EMT were assessed using western blot analysis in 3 types of human RCC cell lines. The results demonstrated that protein levels of $\mathrm{N}$-cadherin and fibronectin were significantly reduced $(\mathrm{P}<0.01$; Fig. 5), and E-cadherin protein levels were significantly increased $(\mathrm{P}<0.01$; Fig. 5) in siCREB-transfected 786-O and OS-RC-2 cells compared with the siNC group, however, the opposite was observed in ACHN cells, which corresponds with the results obtained in wound healing and invasion assays. Furthermore, siCREB transfection did not significantly affect the expression of vimentin compared with the siNC group in all 3 types of RCC cells (Fig. 5). These results suggested that there may be additional mechanisms by which CREB controls RCC function, however, the present study demonstrated that this does not occur via vimentin in vitro, as siCREB transfection did not significantly affect vimentin expression.

The human fibronectin promoter contains a functional CRE sequence. Using bioinformatics software, the authors previously demonstrated that that there is a putative CRE sequence in the promoter of fibronectin, and a putative binding site of CRE-binding protein is found in the promoter of vimentin (21). The present study aimed to determine whether there is a direct interaction between CREB and the CREsite in the fibronectin promoter or the CRE-binding protein site in the vimentin promoter. The current study employed siCREB, to target CREB mRNAs, and ChIP assays with the specific pCREB (Ser133) antibody were performed in 786-O (Fig. 6A), 
A



OS-RC-2
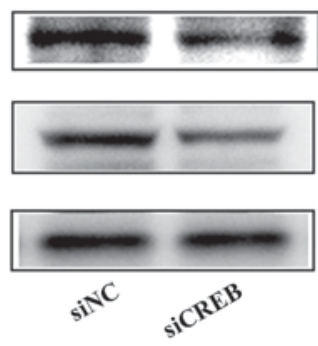

ACHN
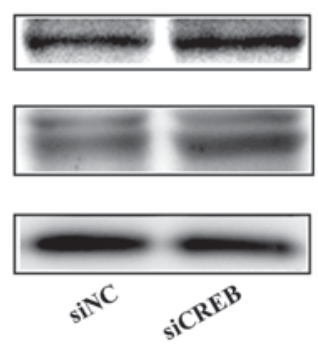
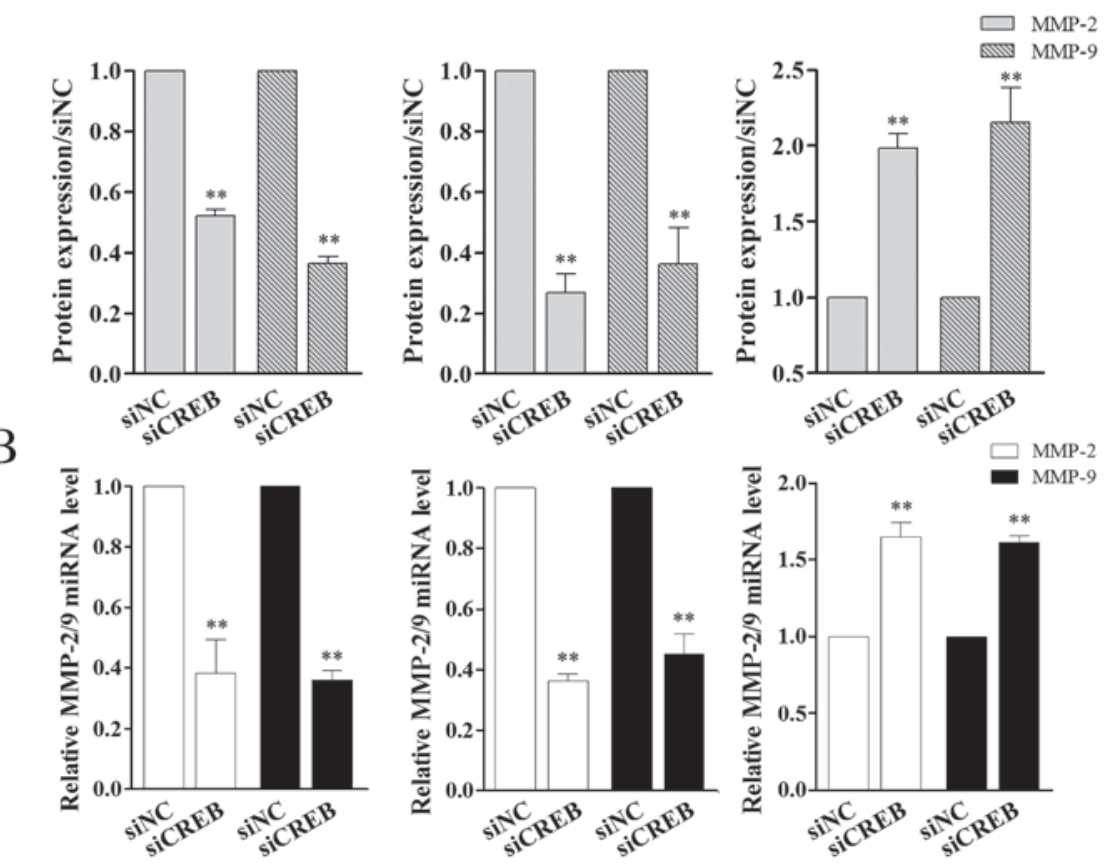

Figure 4. The expression of MMP-2 and MMP-9 protein was controlled by CREB in renal cell carcinoma cell lines. (A) Western blot and (B) quantitative polymerase chain reaction analysis demonstrated that MMP-2 and MMP-9 expression in siNC groups was significantly higher compared with expression in siCREB groups in 786-O and OS-RC-2 cells. In ACHN cells, knockdown of CREB increased MMP-2 and MMP-9 expression. " $\mathrm{P}<0.05$ and ${ }^{* * *} \mathrm{P}<0.01$ vs. siNC group. Results are presented as the mean \pm standard deviation from 3 independent experiments. MMP, matrix metallopeptidase; CREB, cyclic AMP responsive element-binding protein; siRNA, small interfering RNA; siNC, siRNA negative control; siCREB, siRNA targeting CREB.

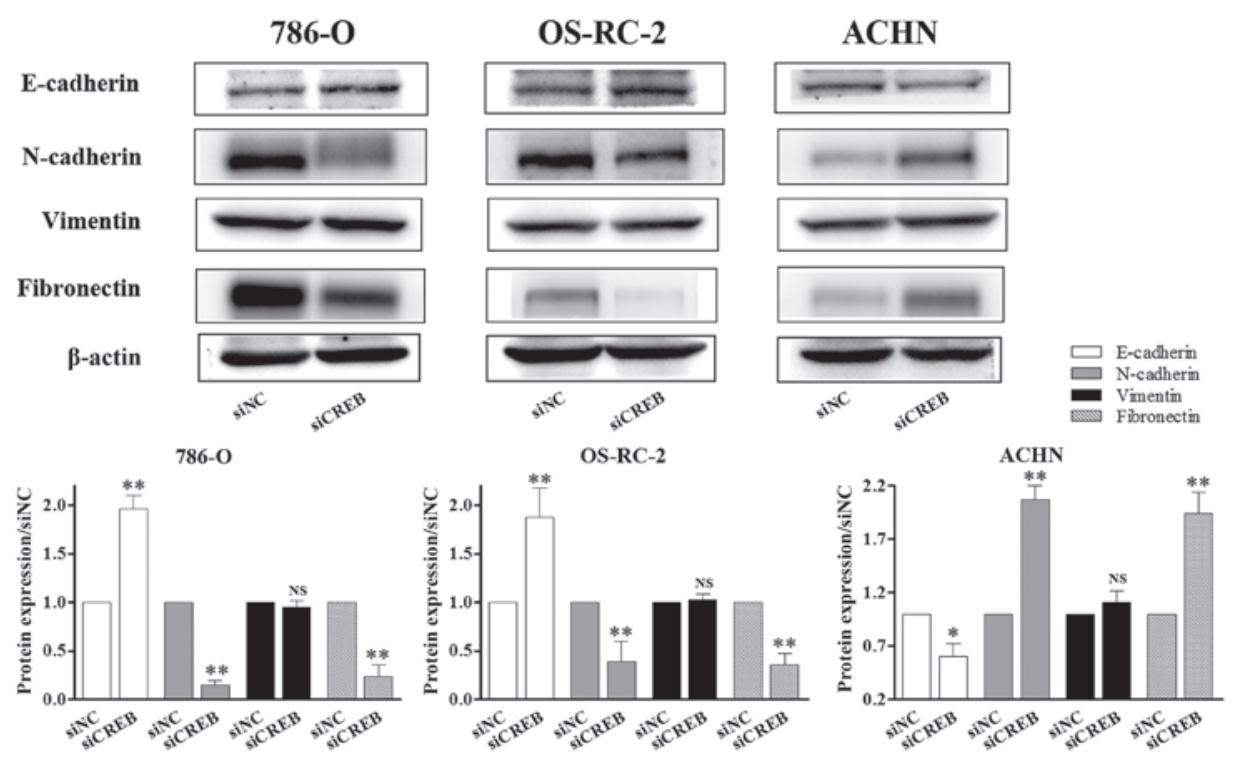

Figure 5. CREB-mediated RCC cell metastasis does not occur via vimentin. RCC cell lines were transiently transfected with siNC or siCREB. The expression of proteins associated with epithelial-mesenchymal transition was determined by western blot analysis. $\mathrm{N}$-cadherin and fibronectin expression was reduced, E-cadherin was increased in 786-O and OS-RC-2 cells compared with the siNC group, however, the opposite results were observed in ACHN cells, and the expression of vimentin was not significantly changed in the 3 types of RCC cells. ${ }^{*} \mathrm{P}<0.05$ and ${ }^{* *} \mathrm{P}<0.01$ vs. siNC group. Results are presented as the mean \pm standard deviation from 3 independent experiments. CREB, cyclic AMP responsive element-binding protein; RCC, renal cell carcinoma; siRNA, small interfering RNA; siNC, siRNA negative control; siCREB, siRNA targeting CREB; NS, not significant. 
A

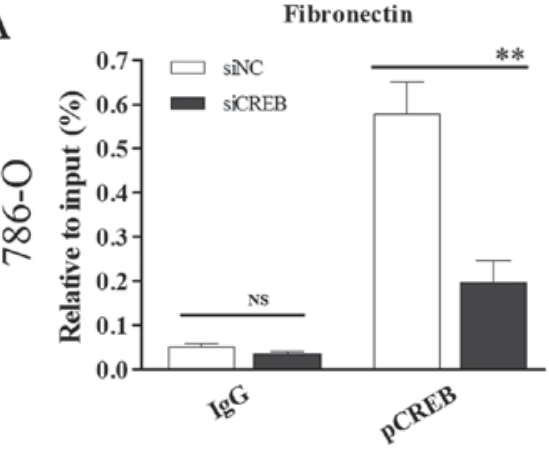

B


Vimentin
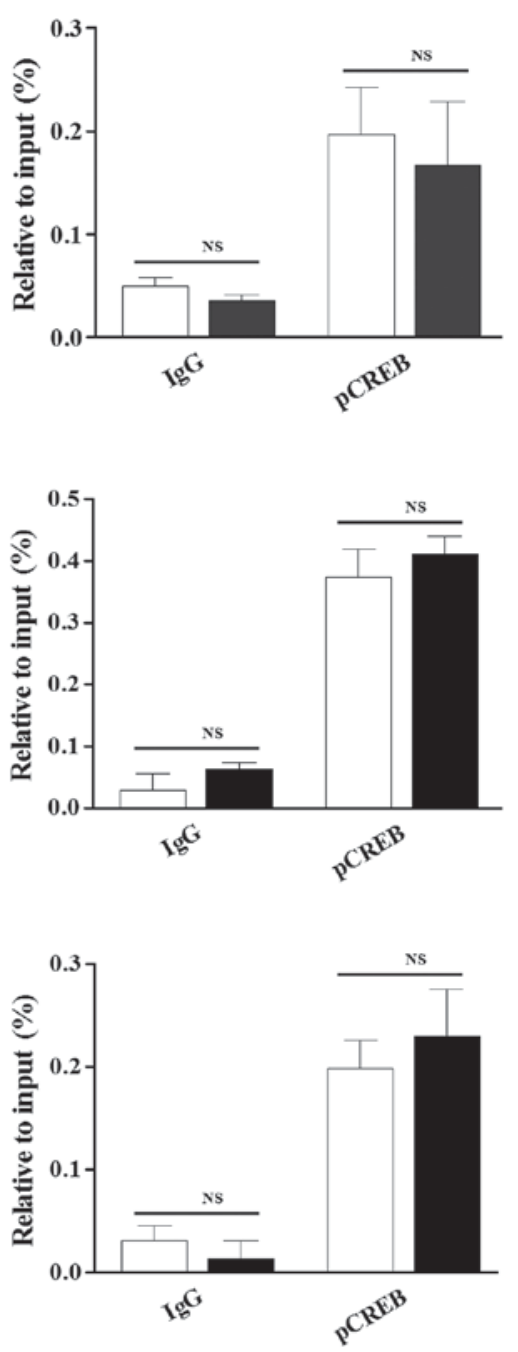

Figure 6. pCREB directly targets fibronectin by binding to its promoter and does not control vimentin. Chromatin immunoprecipitation analysis demonstrated a pCREB interaction with the CRE located at -449 to -442 in the fibronectin promoter and no interaction with the CRE-BP site located at -731 to -722 in the vimentin promoter in (A) 786-O, (B) OS-RC-2 and (C) ACHN cells with specific pCREB (Ser133) antibody. "P $<0.05$ and ${ }^{* *} \mathrm{P}<0.01$ vs. siNC group. Results are presented as the mean \pm standard deviation from 3 independent experiments. CREB, cyclic AMP responsive element-binding protein; pCREB, phosphorylated CREB; CRE, cAMP responsive element; CRE-BP, CRE-binding protein; siRNA, small interfering RNA; siNC, siRNA negative control; siCREB, siRNA targeting CREB; IgG, immunoglobulin G; NS, not significant.
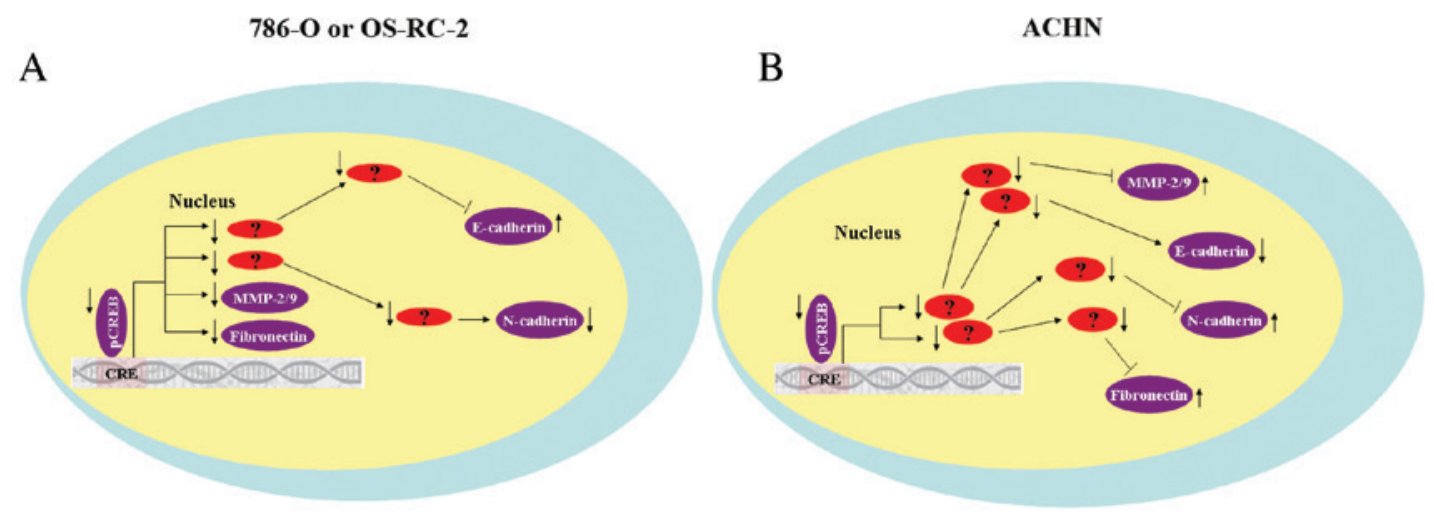

Figure 7. CREB may act through different signaling pathways to regulate the expression of MMP-2/9 and EMT-associated proteins. (A) Proposed molecular mechanisms by which CREB may affect cancer metastasis in human renal cell carcinoma. (A) Knockdown of CREB suppressed the expression of MMP-2/9 and EMT progression, which led to reduced 786-O and OS-RC-2 cell migration and invasion. (B) Knockdown of CREB may activate the expression of MMP-2/9 and EMT progression through an unknown receptor, which may subsequently induce ACHN cell migration and invasion. CREB, cyclic AMP responsive element-binding protein; MMP, matrix metallopeptidase; EMT, epithelial-mesenchymal transition; pCREB, phosphorylated CREB; CRE, cAMP responsive element. 
OS-RC-2 (Fig. 6B) and ACHN (Fig. 6C) cells. Compared with siNC treatment, siCREB treatment significantly decreased pCREB binding to the fibronectin promoter CRE sequence in all 3 cell lines $(\mathrm{P}<0.05$; Fig. $6 \mathrm{~A}-\mathrm{C})$ and did not significantly affect $\mathrm{pCREB}$ binding to the vimentin promoter CRE-binding protein site. The results of this experiment increased evidence for a direct interaction of pCREB (Ser133) with the fibronectin promoter in RCC, whilst confirming that pCREB (Ser133) does not target the vimentin promoter.

\section{Discussion}

CREB has important roles in the regulation of various cellular functions. It is highly expressed and constitutively phosphorylated in a number of types of human cancer (8) and it is widely regarded that $\mathrm{CREB}$ is a proto-oncogenic transcription factor $(8,33)$. However, whether CREB regulates the metastatic potential of RCC cells requires further investigation. The present study aimed to determine the anti-metastatic effect of CREB on human RCC cells by investigating the regulation of MMP-2/9 expression and the potential pathways involved in this regulation.

Invasion and migration are two vital features of metastatic malignancies, and are thought to increase the metastatic potential of cancer cells (34). The present study demonstrated that silencing CREB suppressed the migration and invasion of 786-O and OS-RC-2 cells, whereas it promoted the migration and invasion of ACHN cells, and had no effect in HK-2 cells (Figs. 2 and 3). During metastasis, MMPs facilitate the degradation and invasion of extracellular matrix components, and participate in the onset and progression of tumors $(35,36)$. Park et al (37) demonstrated that intracellular adhesion molecule 3 induced MMP-2 and MMP-9 expression via Akt, and CREB enhanced the migratory and invasive potential of human non-small cell lung cancer cells (37). Other previous studies have identified that, in human osteosarcoma cells, cholangiocarcinoma cells and macrophages, CREB induced expression of MMPs (38-40). The results of the present study further demonstrated that, in 786-O and OS-RC-2 cells, CREB knockdown induced reduced MMP-2/9 expression, as determined by western blot and qPCR analysis. However, CREB silencing increased MMP-2/9 expression in ACHN cells (Fig. 4). The results of western blot and qPCR analysis corresponded with the results of wound healing and invasion assays. The results of the present study indicated that CREB-induced MMP-2/9 expression may be involved in tumor metastasis progression.

EMT has been implicated as a potential mechanism of metastasis (41), it transforms epithelial tumor cells and confers the mesenchymal characteristics that facilitate the dissemination of cells, which subsequently leads to metastasis (42). It has been previously reported that EMT influences RCC progression (43). However, the results of the present study indicate that expression of the mesenchymal markers $\mathrm{N}$-cadherin and fibronectin, and the epithelial marker E-cadherin, were altered as a result of CREB silencing in 786-O and OS-RC-2 cells. However, in ACHN cells, siCREB transfection had the opposite effect on the expression of these proteins (Fig. 5). Furthermore, no changes in the expression of vimentin protein were detected in the 3 types of RCC cells (Fig. 5), which is consistent with the results of our previous study (21). Previously, our laboratory identified putative CRE sites in the promoter of fibronectin, however, only a putative binding site of CRE-BP was identified in the promoter of vimentin (21). Notably, the present study demonstrated the binding of pCREB to the promoter sequence of fibronectin and the absence of pCREB binding to vimentin in 786-O, OS-RC-2 and ACHN cells by ChIP assay (Fig. 6). We hypothesized that the decrease in metastatic potential when CREB was suppressed in 786-O and OS-RC-2 cells is linked to MMP-2/9 and EMT-associated markers, including fibronectin, E-cadherin and $\mathrm{N}$-cadherin. The results of the present study were similar to a report by Cho et al (44), which demonstrated that $\gamma$-ionizing radiation induced increases in CREB-1 led to increased invasion/migration and EMT in non-small cell lung cancer cells (44). However, the regulation of ACHN cell function by $\mathrm{CREB}$ requires further investigation and discussion.

The results of the current study demonstrated that CREB may regulated metastatic RCC cells by mediating expression of MMP-2/9 and EMT-associated proteins, however, results were not consistent between the 3 RCC cell lines. It was hypothesized that there at least two reasons that may explain this phenomenon. Firstly, expression of CREB in the 3 RCC cell lines is different. Compared with normal renal cells (HK-2), expression of total CREB is upregulated in 786-O cells, and is marginally decreased in OS-RC-2 cells and partially downregulated in ACHN cells. In addition, CREB mediated expression of MMP-2/9 and EMT-associated protein may control tumor metastasis via different signaling pathways (Fig. 7). Further studies are required to determine the precise molecular mechanisms by which inhibition of CREB expression advanced cancer cell metastasis, and, conversely, the mechanisms by which the interruption of CREB signaling inhibited cancer cell metastasis.

In conclusion, the present study demonstrated that CREB may act as a critical factor that promotes 786-O and OS-RC-2 cell metastasis by regulating the expression of MMP-2/9 and EMT-associated proteins. It was indicated that this role may be different in ACHN cells, and pCREB did not control vimentin expression in any of the 3 RCC cell lines. Understanding the mechanisms by which CREB affects metastatic RCC via the MMP-2/9 and EMT may aid in the development of novel strategies for the treatment of patients with acquired or innate resistance to cancer treatments.

\section{Acknowledgements}

The current study was supported by grants from Scientific Plan of Medical and Health of Zhejiang Province (grant nos. 2015RCB024 and 2017KY604) and Ningbo Natural Science Foundation (grant no. 2016A610018).

\section{References}

1. Ljungberg B, Campbell SC, Choi HY, Jacqmin D, Lee JE, Weikert S and Kiemeney LA: The epidemiology of renal cell carcinoma. Eur Urol 60: 615-621, 2011.

2. Siegel RL, Miller KD and Jemal A: Cancer statistics, 2015. CA Cancer J Clin 65: 5-29, 2015.

3. Mastoraki A, Mastoraki S, Tsikala-Vafea M, Papanikolaou IS, Lazaris A, Smyrniotis V and Arkadopoulos N: Prognostic benefit of surgical management of renal cell carcinoma invading the inferior vena cava. Indian J Surg Oncol 8: 14-18, 2017. 
4. Fu L, Minton DR, Zhang T, Nanus DM and Gudas LJ: Genome-wide profiling of TRACK kidneys shows similarity to the human ccRCC transcriptome. Mol Cancer Res 13: 870-878, 2015.

5. Liang L, Li L, Zeng J, Gao Y, Chen YL, Wang ZQ, Wang XY, Chang LS and He D: Inhibitory effect of silibinin on EGFR signal-induced renal cell carcinoma progression via suppression of the EGFR/MMP-9 signaling pathway. Oncol Rep 28 999-1005, 2012.

6. Eggener SE, Yossepowitch O, Pettus JA, Snyder ME, Motzer RJ and Russo P: Renal cell carcinoma recurrence after nephrectomy for localized disease: Predicting survival from time of recurrence. J Clin Oncol 24: 3101-3106, 2006.

7. Jean D and Bar-Eli M: Regulation of tumor growth and metastasis of human melanoma by the CREB transcription factor family. Mol Cell Biochem 212: 19-28, 2000.

8. Xiao X, Li BX, Mitton B, Ikeda A and Sakamoto KM: Targeting CREB for cancer therapy: Friend or foe. Curr Cancer Drug Targets 10: 384-91, 2010.

9. Sakamoto KM and Frank DA: CREB in the pathophysiology of cancer: Implications for targeting transcription factors for cancer therapy. Clin Cancer Res 15: 2583-2587, 2009.

10. Shaywitz AJ and Greenberg ME: CREB: A stimulus-induced transcription factor activated by a diverse array of extracellular signals. Annu Rev Biochem 68: 821-861, 1999.

11. Huang S, Ren Y, Wang P, Li Y, Wang X, Zhuang H, Fang R, Wang Y, Liu N, Hehir M and Zhou JX: Transcription factor CREB is involved in CaSR-mediated cytoskeleton gene expression. Anat Rec (Hoboken) 298: 501-512, 2015.

12. Zhuang H, Meng X, Li Y, Wang X, Huang S, Liu K, Hehir M, Fang R, Jiang L, Zhou JX, et al: Cyclic AMP responsive element-binding protein promotes renal cell carcinoma proliferation probably via the expression of spindle and kinetochore-associated protein 2. Oncotarget 7: 16325-16337, 2016.

13. Cho EC, Mitton B and Sakamoto KM: CREB and leukemogenesis. Crit Rev Oncog 16: 37-46, 2011.

14. van der Sligte NE, Kampen KR, ter Elst A, Scherpen FJ, Meeuwsen-de Boer TG, Guryev V, van Leeuwen FN, Kornblau SM and de Bont ES: Essential role for cyclic-AMP responsive element binding protein 1 (CREB) in the survival of acute lymphoblastic leukemia. Oncotarget 6: 14970-14981, 2015.

15. Peng B, Lei N, Chai Y, Chan EK and Zhang JY: CIP2A regulates cancer metabolism and CREB phosphorylation in non-small cell lung cancer. Mol Biosyst 11: 105-114, 2015.

16. Seo HS, Liu DD, Bekele BN, Kim MK, Pisters K, Lippman SM, Wistuba II and Koo JS: Cyclic AMP response element-binding protein overexpression: A feature associated with negative prognosis in never smokers with non-small cell lung cancer. Cancer Res 68: 6065-6073, 2008

17. Singh R, Shankar BS and Sainis KB: TGF- $\beta 1-R O S-A T M-C R E B$ signaling axis in macrophage mediated migration of human breast cancer MCF7 cells. Cell Signal 26: 1604-1615, 2014.

18. Zhang S, Chen L, Cui B, Chuang HY, Yu J, Wang-Rodriguez J, Tang L, Chen G, Basak GW and Kipps TJ: ROR1 is expressed in human breast cancer and associated with enhanced tumor-cell growth. PLoS One 7: e31127, 2012.

19. Liu YL, Lai F, Wilmott JS, Yan XG, Liu XY, Luan Q, Guo ST, Jiang CC, Tseng HY, Scolyer R A, et al: Noxa upregulation by oncogenic activation of MEK/ERK through CREB promotes autophagy in human melanoma cells. Oncotarget 5: 11237-11251, 2014.

20. Kovach SJ, Price JA, Shaw CM, Theodorakis NG and McKillop IH: Role of cyclic-AMP responsive element binding (CREB) proteins in cell proliferation in a rat model of hepatocellular carcinoma. J Cell Physiol 206: 411-419, 2006.

21. Wang X, Ren Y, Zhuang H, Meng X, Huang S, Li Y, Hehir M and Wang P: Decrease of phosphorylated proto-oncogene CREB at Ser 133 site inhibits growth and metastatic activity of renal cell cancer. Expert Opin Ther Targets 19: 985-95, 2015.

22. Zi Y, Zhao W, Zhou J, He H and Xie M: Silencing of TMSG1 enhances metastasis capacity by targeting V-ATPase in breast cancer. Int J Clin Exp Pathol 8: 1312-1320, 2015.

23. Xia M, Yao L, Zhang Q, Wang F, Mei H, Guo X and Huang W: Long noncoding RNAHOTAIR promotes metastasis of renal cell carcinoma by up-regulating histone H3K27demethylase JMJD3. Oncotarget: Feb 3, 2017 (Epub ahead of print).

24. Wang P, Yan H and Li JC: CREB-mediated Bcl-2 expression in trichosanthin-induced Hela cell apoptosis. Biochem Biophys Res Commun 363: 101-105, 2007.
25. Livak KJ and Schmittgen TD: Analysis of relative gene expression data using real-time quantitative PCR and the 2(-Delta Delta C (T)) Method. Methods 25: 402-408, 2001.

26. Jeon SH, Chae BC, Kim HA, Seo GY, Seo DW, Chun GT, Yie SW, Eom SH and Kim PH: The PKA/CREB pathway is closely involved in VEGF expression in mouse macrophages. Mol Cells 23: 23-29, 2007.

27. Khoufache K, Bazin S, Girard K, Guillemette J, Roy MC, Verreault JP, Al-Abed Y, Foster W and Akoum A: Macrophage migration inhibitory factor antagonist blocks the development of endometriosis in vivo. PLoS One 7: e37264, 2012.

28. Shuman Moss LA, Jensen-Taubman S and Stetler-Stevenson WG: Matrix metalloproteinases: Changing roles in tumor progression and metastasis. Am J Pathol 181: 1895-1889, 2012.

29. Hofmann UB, Houben R, Bröcker EB and Becker JC: Role of matrix metalloproteinases in melanoma cell invasion. Biochimie 87: 307-314, 2005.

30. Cheng HL, Hsieh MJ, Yang JS, Lin CW, Lue KH, Lu KH and Yang SF: Nobiletin inhibits human osteosarcoma cells metastasis by blocking ERK and JNK-mediated MMPs expression. Oncotarget 7: 35208-35223, 2016.

31. Melnikova VO, Mourad-Zeidan AA, Lev DC and Bar-Eli M: Platelet-activating factor mediates MMP-2 expression and activation via phosphorylation of cAMP-response element-binding protein and contributes to melanoma metastasis. J Biol Chem 281: 2911-2922, 2006

32. Taparra K, Tran PT and Zachara NE: Hijacking the hexosamine biosynthetic pathway to promote EMT-mediated neoplastic phenotypes. Front Oncol 6: 85, 2016.

33. Kinjo K, Sandoval S, Sakamoto KM and Shankar DB: The role of CREB as a proto-oncogene in hematopoiesis. Cell Cycle 4: 1134-1335, 2005.

34. Ma X, Gu L, Li H, Gao Y, Li X, Shen D, Gong H, Li S, Niu S, Zhang Y, et al: Hypoxia-induced overexpression of stanniocalcin-1 is associated with the metastasis of early stage clear cell renal cell carcinoma. J Transl Med 13: 56, 2015.

35. Li H, Zhang K, Liu LH, Ouyang Y, Bu J, Guo HB and Xiao T: A systematic review of matrix metalloproteinase 9 as a biomarker of survival in patients with osteosarcoma. Tumour Biol 35: 5487-5491, 2014

36. Willis AL, Sabeh F, Li XY and Weiss SJ: Extracellular matrix determinants and the regulation of cancer cell invasion stratagems. J Microsc 251: 250-60, 2013.

37. Park JK, Park SH, So K, Bae IH, Yoo YD and Um HD: ICAM-3 enhances the migratory and invasive potential of human non-small cell lung cancer cells by inducing MMP-2 and MMP-9 via Akt and CREB. Int J Oncol 36: 181-192, 2010.

38. Yang SF, Lee WJ, Tan P, Tang CH, Hsiao M, Hsieh FK and Chien MH: Upregulation of miR-328 and inhibition of CREB-DNA-binding activity are critical for resveratrol-mediated suppression of matrix metalloproteinase- 2 and subsequent metastatic ability in human osteosarcomas. Oncotarget 6: 2736-2753, 2015

39. Sun B, Rong R, Jiang H, Zhang H, Wang Y, Bai X, Zhang M, Ma J, Xia S, Shu W, et al: Prostaglandin E2 receptor EP1 phosphorylate CREB and mediates MMP2 expression in human cholangiocarcinoma cells. Mol Cell Biochem 378: 195-203, 2013.

40. Lee DK, Park EJ, Kim EK, Jin J, Kim JS, Shin IJ, Kim BY, Lee H and Kim DE: Atorvastatin and simvastatin, but not pravastatin, up-regulate LPS-induced MMP-9 expression in macrophages by regulating phosphorylation of ERK and CREB. Cell Physiol Biochem 30: 499-511, 2012.

41. Chaffer CL and Weinberg RA: A perspective on cancer cell metastasis. Science 331: 1559-1564, 2011.

42. Ni D, Ma X, Li HZ, Gao Y, Li XT, Zhang Y, Ai Q, Zhang P, Song EL, Huang QB, et al: Downregulation of FOXO3a promotes tumor metastasis and is associated with metastasis-free survival of patients with clear cell renal cell carcinoma. Clin Cancer Res 20: 1779-1790, 2014.

43. O'Mahony FC, Faratian D, Varley J, Nanda J, Theodoulou M, Riddick AC, Harrison DJ and Stewart GD: The use of automated quantitative analysis to evaluate epithelial-to-mesenchymal transition associated proteins in clear cell renal cell carcinoma. PLoS One 7: e31557, 2012.

44. Cho JH, Hong WG, Jung YJ, Lee J, Lee E, Hwang SG, Um HD and Park JK: Gamma-Ionizing radiation-induced activation of the EGFR-p38/ERK-STAT3/CREB-1-EMT pathway promotes the migration/invasion of non-small cell lung cancer cells and is inhibited by podophyllotoxin acetate. Tumour Biol 37: 7315-7325, 2016. 